\title{
ACCURACY OF ESTIMATES OF VOLUME FRACTION
}

\author{
JOANNE CHIA AND ADRIAN BADDELEY
}

Department of Mathematics and Statistics University of Western Australia Nedlands, W.A. 6907 Perth, Australia e-mail: chia@maths.uwa.edu.au

(Accepted August 3, 2000)

\begin{abstract}
When estimating a volume fraction $V_{V}$ from point count fractions $P_{P}$ using Delesse's principle $V_{V}=P_{P}$, very little information on the accuracy of the estimator can be obtained from the basic theory of stereology. Existing methods for quantifying the variability of $P_{P}$ are mainly large-sample approximations such as Cochran's formula for the variance of a ratio. Cruz-Orive proposed an alternative method, but this requires statistical assumptions to be made on the point counts $\mathrm{P}$, that do not hold in general. We introduce two alternative methods for quantifying the variability of $P_{P}$, namely the bootstrap method and explicit statistical modelling of the bivariate distribution. The bootstrap method requires few statistical assumptions about the point counts but requires large sample size. The explicit statistical modelling method does make assumptions, but they can be checked directly from the data. To explore this approach, we propose a statistical model, the Type I Bivariate Binomial (BVB) distribution to model the pairs of count data $(P, P)$. We show how to fit the BVB model to the data and how to assess the goodness-of-fit of this model. A formula for the variance of $P_{P}$ under the BVB model is also derived. The three approaches are compared in their application to nine example data sets taken from macroscopic sections of cerebral hemispheres of selected domesticated animals. The BVB model appears to be a good fit to these data sets. Implications for stereological estimation are discussed.
\end{abstract}

Keywords: bootstrap method, delta method, Monte Carlo, stereology, type I bivariate binomial distribution, volume fraction.

\section{INTRODUCTION}

Many parameters of interest in stereology are ratios of geometrical quantities such as the volume fraction

$$
V_{V}=\frac{V(\text { obj })}{V(\text { ref })}
$$

of a phase of interest, obj within the threedimensional reference space, ref. Stereological theory (Weibel, 1980; Baddeley, 1991), shows how to statistically estimate $V(\mathrm{obj})$ and $V(\mathrm{ref})$ by unbiased estimators, such as the Cavalieri's principle estimators

and

$$
\text { est } V(\mathrm{obj})=t . a \sum P(\mathrm{obj})
$$

$$
\operatorname{est} V(\text { ref })=t \cdot a \sum P(\text { ref })
$$

where $\mathrm{a}$ is the area per test point and $\mathrm{t}$ is the separation distance between the sections, based on test point counts $P(\mathrm{obj})$ and $P(\mathrm{ref})$. Usually, the ratio $V_{V}$ is estimated by taking the ratios of these estimators,

$$
\text { est } V_{V}=\frac{\text { est } V(\text { obj })}{\text { est } V(\text { ref })}=\frac{\sum P(\text { obj })}{\sum P(\text { ref })} .
$$

The important question for us is the accuracy of this estimator. Stereological theory (Weibel, 1980) does not provide much information about the variance of the individual estimators est $V($ obj), est $V$ (ref) or their ratio.

In a more general context, suppose we are interested in the parameter

$$
\theta=\frac{\mathrm{E}(\mathrm{Y})}{\mathrm{E}(\mathrm{X})}
$$

where $X$ and $Y$ are geometrical measurements such as length, area or the number of counts of test points within a small planar section through an object. The parameter $\theta$ is usually estimated by the ratio estimator

$$
\hat{\theta}=\frac{\sum y_{i}}{\sum x_{i}}=\frac{\bar{y}}{\bar{x}} .
$$


Our aim is to estimate the variance, $\operatorname{Var}(\hat{\theta})$ of this estimator.

The usual estimator of $\theta$ used in stereology is the ratio estimator (2) which does not require any specific model assumptions. Cruz-Orive (1980) proposed a semi-parametric approach to estimate $\theta$, and in this paper, we use a parametric approach. We consider bivariate count data $(X, Y)$ and the situation where $n$ integer bivariate observations $\left(x_{l}, y_{l}\right), \ldots,\left(x_{n}\right.$, $\left.y_{n}\right)$, are obtained from $\mathrm{n}$ different images or sections of the material under study. These observations are assumed to be independent and to have the same bivariate distribution.

\section{EXAMPLE DATA SETS}

The data were extracted from a comparative study of neuroanatomy by Mayhew et al., (1990) and were kindly provided by Professor Mayhew. The samples were taken from the cerebral hemispheres of nine selected domesticated animals, namely three horses, one dog, one pig and four rabbits.

A test system consisting of a grid of test points and a lattice of cycloid arcs was used to record various quantities of interest for the study. Volume fraction of cortex in the whole brain was estimated from $V_{V}($ cort $/$ ref $)=P($ cort $) / P($ ref $)$ where "cort" represents cortex. In this paper, we denote $P($ ref $)$ the number of test points which fell on the whole slice by $X$, and $P($ cort $)$ the number of test points which fell on the cortex alone by $Y$.

\section{VARIANCE ESTIMATION}

We consider three approaches to estimate the variance of $\hat{\theta}=\bar{y} / \bar{x}$. They are the non-parametric, semi-parametric and parametric approaches.

\section{Bootstrap method}

A non-parametric bootstrap method (Efron, 1982) was applied to each of the nine data sets. For each data set, 1000 random samples (with replacement) from the data, of the same size as the original sample, were generated and the sample value of $\hat{\theta}=\bar{y} / \bar{x}$ computed. The variance of $\hat{\theta}$ was estimated by the sample variance of the bootstrap replications.

\section{Delta/Cochran method}

One may apply the delta method (Lehmann, 1999 , p. 85) which assumes that $(\bar{X}, \bar{Y})$ are asymptotically jointly normally distributed and approximates the variance of $\hat{\theta}$ by linearising the function $f(x, y)=y / x$ around $(\mathrm{E}(\bar{X}), \mathrm{E}(\bar{Y}))$. This yields an estimate of the variance of $\operatorname{Var}(\hat{\theta}) \approx \frac{1}{n}\left[\left(\frac{\bar{y}^{2}}{\bar{x}^{4}}\right) s_{x}^{2}+\left(\frac{1}{\bar{x}^{2}}\right) s_{y}^{2}-2\left(\frac{\bar{y}}{\bar{x}^{2}}\right)\left(\frac{1}{\bar{x}}\right) s_{x y}\right]$,

where $s_{x}^{2}, s_{y}^{2}$ and $s_{x y}$ denote the sample variance of $x$ and of $y$ and the sample covariance of $x$ and $y$ respectively. Note that the above estimate of the variance of $\hat{\theta}$ is equivalent to the one given by Cochran (1977).

\section{Cruz-Orive method}

The semi-parametric approach adopted to estimate the variance of $\hat{\theta}$ was that proposed by Cruz-Orive (1980). This method is based on a conditional regression model which assumes linear regression of $y$ on $x$ and the variance of $y$ given $x$ is proportional to $x$. Under this model,

$$
\operatorname{Var}(\hat{\theta}) \simeq \frac{1}{n-1}\left[\sum_{i=1}^{n}\left(\frac{y_{i}^{2}}{x_{i}}\right) / \sum_{i=1}^{n} x_{i}-\hat{\theta}^{2}\right] .
$$

\section{Parametric method}

For the parametric approach, we assume that the statistical model, Type I Bivariate Binomial distribution (BVB) is applicable to the data. Details of the BVB model are given in a further section below. We fitted this model to the data and used the variance of $\hat{\theta}$ predicted by the model. The following expression for the variance of $\theta$ was derived from the BVB model where $P_{A}$ and $P_{X}$ are parameters of the distribution (see below), $n$ is the number of sections/fields and $m$ is the number of test points on the test system.

$$
\operatorname{Var}(\hat{\theta}) \simeq \frac{P_{A}}{P_{X}}\left(1-P_{A} / P_{X}\right)\left\{(1-2 / n m)\left[n m \cdot P_{X}-\left(1-P_{X}\right)\right]\right\}(4)
$$

\section{RESULTS}

We applied the techniques of estimating the variance of $\hat{\theta}$ mentioned above to the nine example data sets. The estimates of the standard error of $\hat{\theta}$ obtained are shown in Table 1 and Fig. 1. The bootstrap method, delta/Cochran method and CruzOrive's formula yielded results that are roughly in agreement with each other for all the data sets, while they disagree with the estimates obtained by the BVB 
model, particularly for the rabbit data (R2, R3, R4) which have small sample size. Further, we can see from Fig. 1 that for data sets R2, R3, R4, the bootstrap, delta/Cochran and Cruz-Orive methods produced estimates that fluctuate more than those yielded by the BVB model.

A simulation study was then carried out to investigate the variance of the estimates of $\operatorname{Var}(\hat{\theta})$ obtained by the four techniques assuming the BVB model is true. For a particular data set, we simulated 1000 matching data sets from the BVB model using the maximum likelihood estimates of the parameters. The four methods of estimating $\operatorname{Var}(\hat{\theta})$ were then applied to the simulated data, and the corresponding sample variance/covariance matrix was computed.
The nine variance/covariance matrices obtained all showed that the variance of the estimates of $\operatorname{Var}(\hat{\theta})$ obtained by the BVB model is much lower than those yielded by the bootstrap, delta and Cruz-Orive methods. As an example, the variances for data set H1 were $3.6 \times 10^{-7}$ for the BVB model, $7.4 \times 10^{-6}$ for the bootstrap method, $4.4 \times 10^{-6}$ for the delta/Cochran method and $3.8 \times 10^{-6}$ for Cruz-Orive method. In addition, it was found that the latter three estimates were highly correlated.

The results obtained from the four variance estimation methods and the simulation study indicate that if we believe the BVB model is true for our data, then it provides the most reliable way to estimate $\operatorname{Var}(\hat{\theta})$ amongst the four methods considered.

\section{Estimates of standard error}

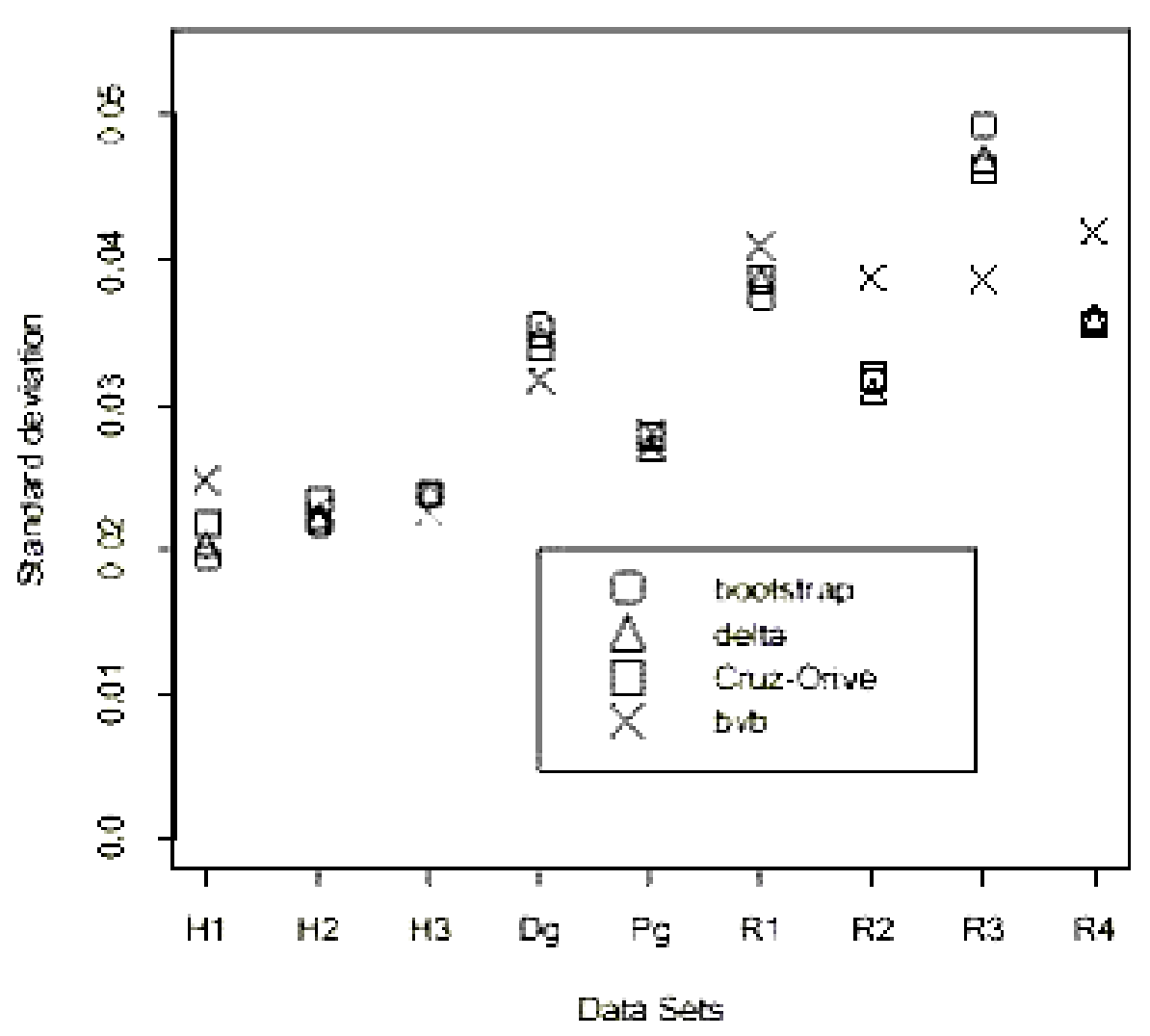

Fig. 1. Estimates of standard error of $\hat{\theta}$ using the bootstrap method, delta method, Cruz-Orive's formula and BVB model. 
Table 1. Estimates of standard error of $\hat{\theta}=\bar{y} / \bar{x}$ using the bootstrap, delta/Cochran, Cruz-Orive methods and the BVB model(4).

\begin{tabular}{llllllll}
\hline & & & \multicolumn{5}{l}{ Estimated Standard Error } \\
\cline { 5 - 7 } $\begin{array}{l}\text { Data } \\
\text { Set }\end{array}$ & Species & $\begin{array}{l}\text { Sample } \\
\text { size }\end{array}$ & $\begin{array}{l}\hat{\theta} \\
\text { values }\end{array}$ & $\begin{array}{l}\text { Bootstrap } \\
\text { Method }\end{array}$ & $\begin{array}{l}\text { Delta } \\
\text { Method }\end{array}$ & $\begin{array}{l}\text { Cruz-Orive's } \\
\text { Formula }\end{array}$ & $\begin{array}{l}\text { BVB } \\
\text { Model }\end{array}$ \\
\hline H1 & Horse & 72 & 0.532 & 0.019 & 0.020 & 0.022 & 0.025 \\
H2 & Horse & 76 & 0.485 & 0.022 & 0.022 & 0.023 & 0.023 \\
H3 & Horse & 68 & 0.400 & 0.024 & 0.024 & 0.024 & 0.022 \\
Dg & Dog & 64 & 0.602 & 0.035 & 0.035 & 0.034 & 0.032 \\
Pg & Pig & 68 & 0.470 & 0.028 & 0.027 & 0.028 & 0.028 \\
R1 & Rabbit & 40 & 0.640 & 0.037 & 0.038 & 0.039 & 0.041 \\
R2 & Rabbit & 32 & 0.590 & 0.032 & 0.031 & 0.032 & 0.039 \\
R3 & Rabbit & 40 & 0.592 & 0.049 & 0.047 & 0.046 & 0.039 \\
R4 & Rabbit & 36 & 0.496 & 0.036 & 0.036 & 0.035 & 0.042 \\
\hline
\end{tabular}

\section{TYPE I BIVARIATE BINOMIAL DISTRIBUTION}

The Type I bivariate binomial distribution (BVB) (Kocherlakota and Kocherlakota, 1992) is the joint, distribution of two random variables $X$ and $Y$ with the following joint probability mass function

$$
P(X=x, Y=y)=\sum_{a=\max (0, x+y-m)}^{a=\min (x, y)} \frac{m !}{a !(x-a) !(y-a) !(m-x-y+a) !} P_{A}^{a} P_{B}^{x-a} P_{C}^{y-a} P_{D}^{m-x-y+a},
$$

where $0 \leq P_{A}<1,0 \leq P_{B}<1,0 \leq P_{C}<1,0 \leq P_{D}<1,0 \leq x \leq m$ and $0 \leq y \leq m$.

\section{Parameter Estimation}

We derive the optimal estimator $\hat{\theta}$ of $\theta$ from the BVB model using the identity given by (1). Let $P_{X}=P_{A}+P_{\mathrm{B}}$ and $P_{Y}=P_{A}+P_{C}$. From (Kocherlakota and Kocherlakota, 1992, p. 61), $\mathrm{E}(X)=m P_{X}$ and $\mathrm{E}(Y)=m P_{Y}, \quad$ where the maximum likelihood estimators of $P_{X}$ and $P_{Y}$ given in (Kocherlakota and Kocherlakota, 1992, p. 80) are $\hat{P}_{X}=\bar{x} / m$ and $\hat{P}_{Y}=\bar{y} / m$ respectively with $\bar{x}=\frac{1}{n} \sum_{i=1}^{n} x_{i}$ and $\bar{y}=\frac{1}{n} \sum_{i=1}^{n} y_{i}$. Therefore, $\hat{\theta}=\bar{y} / \bar{x}$, which is the ratio estimator (2).

\section{Motivation}

We consider the case when $\mathrm{P}_{C}=0$. The BVB model can be considered as arising from a sequence of $\mathrm{m}$ bivariate Bernoulli trials with a pair of characteristics being studied at each trial (Kocherlakota and Kocherlakota, 1992). Suppose we have some biological material consisting of empty spaces and two compartments, Tissue I and Tissue II such that Tissue II is contained in Tissue I. Fig. 2 shows a sketch of such a material, upon which a test grid is superimposed.

If a test point falls on Tissue II, then it must have also fallen onto Tissue I, but the converse is not necessarily true. That is, each test point on the test grid can be treated as a bivariate trial with 'success' being that the test point hits Tissue I or Tissue II as appropriate. If the different bivariate trials at different test points can be regarded as independent then the BVB model can be applied here with $\mathrm{P}_{C}$, the probability that a test point is not on Tissue I but is on Tissue II, equal to 0 and $m$, the number of trials, taken as the number of test points on the test grid. 


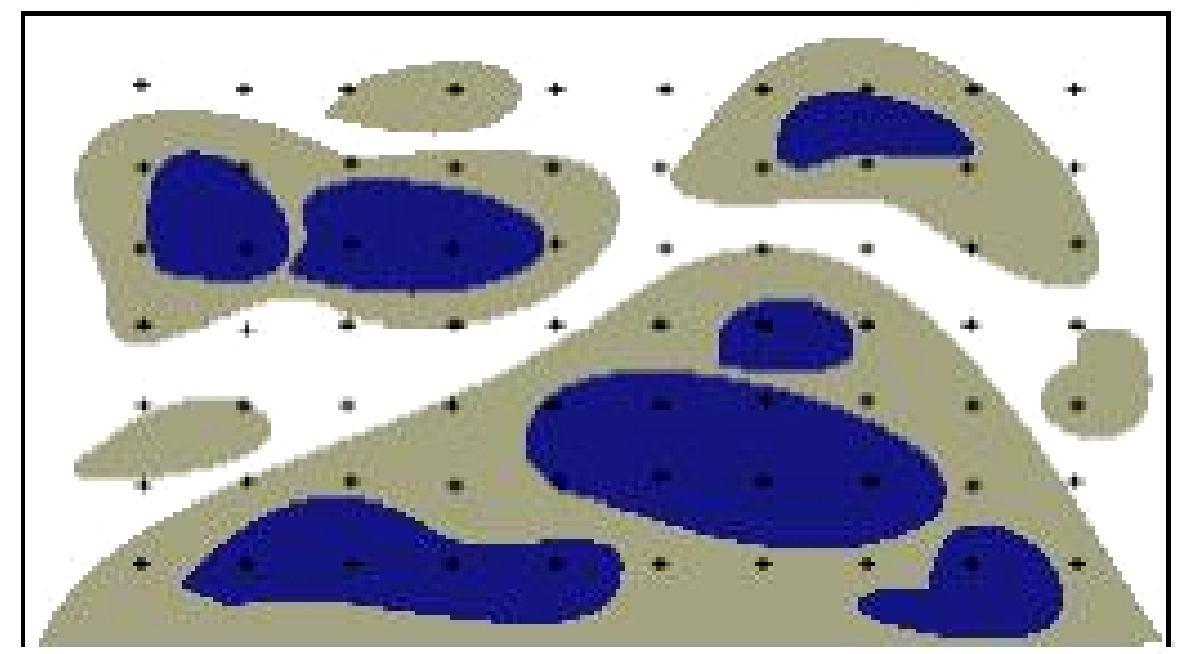

Fig. 2. Sketch of biological material with Tissue II (darker region) contained in Tissue I (lighter region) where “+” indicates a test point on the test grid and white region represents empty spaces.

\section{Applying BVB to the data}

The scheme described above can be applied to the $(X, Y)$ data if we treat "whole slice" as Tissue I and "cortex" as Tissue II. In our case where $\mathrm{P}_{C}=0$, the probability mass function (5) becomes

$P(X=x, Y=y)=\frac{m !}{y !(x-y) !(m-x) !}\left(P_{A}^{y} P_{B}^{x-y} P_{D}^{m-x}\right)$.

To fit this model to the data, we estimate the probabilities using maximumlikelihood. The maximum likelihood estimators found are $\hat{P}_{A}=\bar{y} / m, \hat{P}_{B}=(\bar{x}-\bar{y}) / m$ and $\hat{P}_{D}=1-\bar{x} / m$.

\section{Testing the goodness-of-fit of the BVB model to the data}

To assess the adequacy of the BVB model to the data, we perform a goodness-of-fit test of the BVB distribution against a completely unspecified alternative at the 5\% significance level using a Monte Carlo test (Hope, 1968; Besag and Diggle, 1977). Following (Brooks et al., 1997) we use the maximum log-likelihood as the test statistics; this choice is justified by its connection to the likelihood ratio test.

For each data set, we first fit the BVB model by maximum likelihood, yielding parameter estimates $\hat{P}_{A}, \quad \hat{P}_{B}$ and $\hat{P}_{D}$. Then we generate 99 simulated datasets, of the same size as the original dataset, drawn from the BVB distribution with these parameters. For each simulated dataset the BVB model is fitted by maximum likelihood, and the maximised value of the log-likelihood is recorded. The log-likelihood values from the 99 simulated datasets, and the value for the original data, are pooled and ranked in ascending order. The null hypothesis will be rejected at significance level $2 k / 100$ if the original data value of the maximum loglikelihood is one of the $k$ largest or $k$ smallest values. We set $k=2$.

The simulation test procedure described above is applied to each of the nine data sets. The ranks and the Monte-Carlo p-values of the tests obtained are shown in Table 2 from which we can see that all data sets except that of R4 (marked with an asterisk) result in accepting the BVB model.

Table 2: The ranks and p-values obtained from the Monte Carlo tests for the nine data sets.

\begin{tabular}{ccc}
\hline Data & Rank & p-value \\
\hline H1 & 78 & 0.23 \\
H2 & 2 & 0.99 \\
H3 & 94 & 0.07 \\
Dg & 34 & 0.67 \\
Pg & 26 & 0.75 \\
R1 & 85 & 0.16 \\
R2 & 94 & 0.07 \\
R3 & 8 & 0.93 \\
R4 & 96 & $* 0.05$ \\
\hline
\end{tabular}

To determine whether the BVB model can be considered as an appropriate model for the data from the simulation tests results, we assume that the results 
for each dataset are independent. Then the probability of observing at least one rejection of $H_{0}$ when $H_{0}$ is true in each case is $\mathrm{P}(\operatorname{Bin}(9,0.05) \geq 1)=0.37$ which is not significant, where "Bin" represents the binomial distribution. Hence we conclude that the BVB model is applicable to $(P, P)$ data in this context.

A preliminary report of some of the data was presented at the $\mathrm{X}^{\text {th }}$ International Congress for Stereology, Melbourne, Australia, 1-4 November 1999.

\section{ACKNOWLEDGEMENTS}

We thank Professors T.M. Mayhew, G.L.M. Wamengele, and V. Dantzer for providing us with the stereological data and Dr U. Hahn for valuable feedback.

\section{REFERENCES}

Baddeley A (1991). Stereology. In: Spatial statistics and digital image analysis. Ch. 10. Washington D.C: National Academy Press, 181-216.
Besag J, Diggle, P. (1977). Simple Monte Carlo tests for spatial pattern. Appl Statistics 26:327-33.

Brooks S, Morgan B, Ridout M, Pack S (1997). Finite mixture models for proportions. Biometrics 53:1097115.

Cochran W (1977). Sampling Techniques. New York: Wiley and Sons, $3^{\text {rd }}$ edition.

Cruz-Orive L (1980). Best linear unbiased estimators for stereology. Biometrics 36:595-605.

Efron B (1982). The Jackknife, the Bootstrap and Other Resampling Plans. Society for Industrial and Applied Maths, Philadelphia, Pennsylvania.

Hope A (1968). A simplified Monte Carlo significance test procedure. J Roy Stat Soc 30(B):582-98.

Kocherlakota S, Kocherlakota K (1992). Bivariate Discrete Distributions. New York: Marcel Dekker.

Lehmann E (1999). Elements of large sample theory. New York, USA: Springer-Verlag.

Mayhew T, Wamengele G, Dantzer V (1990). Comparative morphometry of the brain: estimates of cerebral volumes and cortical surface areas obtained from macroscopic slices. J Anatomy 172:191-200.

Weibel E (1980). Stereological Methods. 2. Theoretical Foundations. London: Academic Press. 